\title{
Different Structural Alterations in Individual Conduit Arteries of SHRs Compared to Wistar Rats From the Prehypertensive Period to Late Adulthood
}

\author{
F. KRISTEK ${ }^{1}$, M. DROBNA ${ }^{1}$, S. CACANYIOVA ${ }^{1}$ \\ ${ }^{1}$ Institute of Normal and Pathological Physiology of the Slovak Academy of Sciences, Bratislava, \\ Slovak Republic
}

Received June 15, 2017

Accepted August 18, 2017

\begin{abstract}
Summary
Structural changes of thoracic aorta (TA), carotid (CA) and iliac artery (IA) were assessed in Wistar and spontaneously hypertensive rats (SHR) aged 3, 17, and 52 weeks. Systolic blood pressure (sBP) was measured by plethysmography weekly. After perfusion fixation the arteries were processed for electron microscopy. The wall thickness (WT), cross-sectional area (CSA), inner diameter (ID), and WT/ID in all arteries and volume densities of endothelial cells (ECS), muscle cells (SMCS), and extracellular matrix (ECM) in TA were measured and their CSAs were calculated. In 3-week-old SHR compared to Wistar rats, SBP did not differ; in the TA, all parameters (WT, CSA, ID, WT/ID, CSA of SMCS, CSA of ECS, and CSA of ECM) were decreased; in CA, WT and CSA did not differ, ID was decreased, and WT/ID was increased; in IA, WT, CSA, and ID were increased. In 17- and 52-week-old SHRs, SBP and all parameters in all arteries were increased, only ID in IE in 52-week-old SHRs and CSA of ECs in the TA in 17-week-old SHRs did not change. Disproportionality between BP increase and structural alterations during ontogeny in SHR could reflect the flexibility of the arterial tree to the different needs of supplied areas.
\end{abstract}

\section{Key words}

Ontogeny - Hypertension - Conduit arteries • Geometry • Structure

\section{Corresponding author}

F. Kristek, Sienkiewiczova 1, 81371 Bratislava, Slovak Republic. Fax: +4212552968 516. E-mail: Frantisek.kristek@savba.sk

\section{Introduction}

The efficiency of the cardiovascular system depends on the coordination of functional abilities and structural composition of the heart and particular sections of the arterial tree as a conduit and muscular and resistant arteries. During the age some peculiar pathological changes were revealed in spontaneously hypertensive rats (SHRs). They gradually enhance and contribute to the impairment of the cardiovascular system in the later age period. Structural modifications of the arterial wall significantly affect mechanical properties and functional effectiveness - i.e. the range of constriction and relaxation ability of the respective arteries to vasoactive signals and vice versa. They also determine the amplitude of pressure waves, as well as their propagation and reflection along the arterial tree (O'Rourke and Safar 2005, Cosson et al. 2007, Avolio et al. 2010, Sviglerova et al. 2010). Finally, they can result in an insufficient supply of the particular areas with nutrients. The beginning and causation of these alterations is not exactly established. There is a relative consensus that the main alteration in SHRs such as blood pressure (BP) increase and changes in the heart and arterial wall trophicity and function started from the about the $5^{\text {th }}$ week of age. However, some authors have reported significant alterations in the cardiovascular system of SHRs compared with those in normotensive rats earlier in the prehypertension period (Dickhout and Lee 1997). Nevertheless, there is a discrepancy regarding whether the structural and functional alterations in the heart and arterial wall of conduit arteries start simultaneously with 
BP increase or whether the development of individual changes occur in consecutive order and whether they are uniform and with the same intensity in all arteries. Without answering these questions, the conclusions of studies based on the functional or structural investigation of only one type of artery often in various phases of ontogenic development and applying them to the other arteries or even to the whole cardiovascular system may lead to misinterpretation.

In the present study, we determined early pathological alterations in (i) systolic blood pressure, (ii) geometry, and (iii) structure of the walls of the thoracic aorta, carotid, and iliac artery of SHRs (in three ontogeny periods) from the prehypertensive age to adulthood and late adulthood.

\section{Methods}

Procedures were performed in accordance with institutional guidelines and were approved by the State Veterinary and Food Administration of the Slovak Republic and by an Ethical committee according to the European Convention for the Protection of Vertebrate Animals used for Experimental and other Scientific Purposes, Directive 2010/63/EU of the European Parliament. All rats were housed under a $12 \mathrm{~h}$ light-12 h dark cycle, at a constant humidity (45-65\%) and temperature $\left(20-22{ }^{\circ} \mathrm{C}\right)$, with free access to standard laboratory rat chow and drinking water. The Institute of Normal and Pathological Physiology provided veterinary care.

Three groups (ten animals in each group) of Wistar rats and age-matched SHRs aged 3, 17, and 52 weeks were used for the study. Systolic blood pressure (sBP) was measured non-invasively in pre-warmed animals using the plethysmographic method on the tail artery each week from 3 to 17 weeks of age, and then at the end of the experimental age. Body weight was recorded at the same time. The geometry of the thoracic aorta and carotid and iliac arteries, as well as the structure of the thoracic aorta, was assessed at 3, 17, and 52 weeks of age. At the end of the experiment, the animals were sacrificed by an overdose of anaesthesia using ketamine $(0.25 \mathrm{ml} / 100 \mathrm{~g}$ b.w.) and xylazine $(0.1 \mathrm{ml} / 100 \mathrm{~g}$ b.w. $)$ (Zentiva, Czech Republic) administered i.p. The chest was opened, a cannula was placed into the left ventricle, and the cardiovascular system was perfused at a constant pressure of $90 \mathrm{~mm} \mathrm{Hg}$ (3-week-old rats) and $120 \mathrm{~mm} \mathrm{Hg}$ (the rest of the groups) for 10 min with a fixative solution
(300 $\mathrm{mM}$ glutaraldehyde in $100 \mathrm{mM}$ phosphate buffer). The middle part of the thoracic aorta, iliac, and carotid artery were excised, cut to three segments, and immersed in the same fixative overnight, post-fixed with $40 \mathrm{mM}$ $\mathrm{OsO}_{4}$ in $100 \mathrm{mM}$ phosphate buffer, stained en block with $1 \%$ uranylacetate, dehydrated by a graded alcohol series and propylene oxide, and embedded in Durcupan ACM. Semi-thin sections from two randomly selected blocks were cut perpendicularly to the long axis and were stained with methylene blue. Both the wall thickness (tunica intima + tunica media) and length of inner circumference were measured by light microscopy. The wall thickness was measured at $45^{\circ}$ intervals around the circumference of the artery and from the measured data mean value was calculated. The inner diameter, crosssectional area (tunica intima + tunica media), and wall thickness/inner diameter ratio were evaluated from these data. Thoracic aorta sections of approximately 70-nm thickness were cut on an ultramicrotome (Reichert Nova), stained with alkaline lead citrate and examined in a transmission electron microscope (Tesla BS 500). The volume densities (proportional representation) of endothelial cells (ECs), smooth muscle cells (SMCs), and extracellular matrix (ECM) in the tunica intima and media were determined by the Weibel et al. (1966) point counting method in electron microscopy. In short, the grid was placed on the respective section randomly and 5,000 points were counted. Three sections from two randomly selected blocks from vessels of control and SHR were processed in the same way (together 30,000 points were counted from each animal). From the volume densities, the areas of the ECs, SMCs, and ECM were counted. All of the results were expressed as means \pm S.E.M. Measures were tested for significant differences with the unpaired Student's t-test. A $p<0.05$ was considered to indicate statistical significance.

\section{Results}

\section{Blood pressure values}

In 3-week-old SHRs, the value of sBP $(84.2 \pm 1.4 \mathrm{~mm} \mathrm{Hg})$ did not differ from that of age-matched Wistar rats $(83 \pm 1.9 \mathrm{~mm} \mathrm{Hg})$. In the following weeks, the sBP of both SHRs and Wistar rats continuously increased, but the increment was steeper in SHRs. At the end of the $9^{\text {th }}$ week in age, the sBP was significantly higher in SHRs $(154 \pm 1.4 \mathrm{~mm} \mathrm{Hg})$ than in Wistar rats $107 \pm 1.0 \mathrm{~mm} \mathrm{Hg}$ ). The SBP in 17-week-old SHRs $(214 \pm 7.0 \mathrm{~mm} \mathrm{Hg})$ continued to be elevated, and it 
was significantly increased $(\mathrm{p}<0.01)$ compared with that in age-matched Wistar rats $(114 \pm 1.4 \mathrm{~mm} \mathrm{Hg})$. In 52-week-old SHRs, the SBP $(190 \pm 2.4 \mathrm{~mm} \mathrm{Hg})$ was elevated compared with that in age-matched Wistar rats
$(115 \pm 3.0 \mathrm{~mm} \mathrm{Hg})$. Nevertheless, its absolute value was lower than that in 17-week-old SHR. The changes in the sBP during ontogeny are demonstrated in Figure 1.

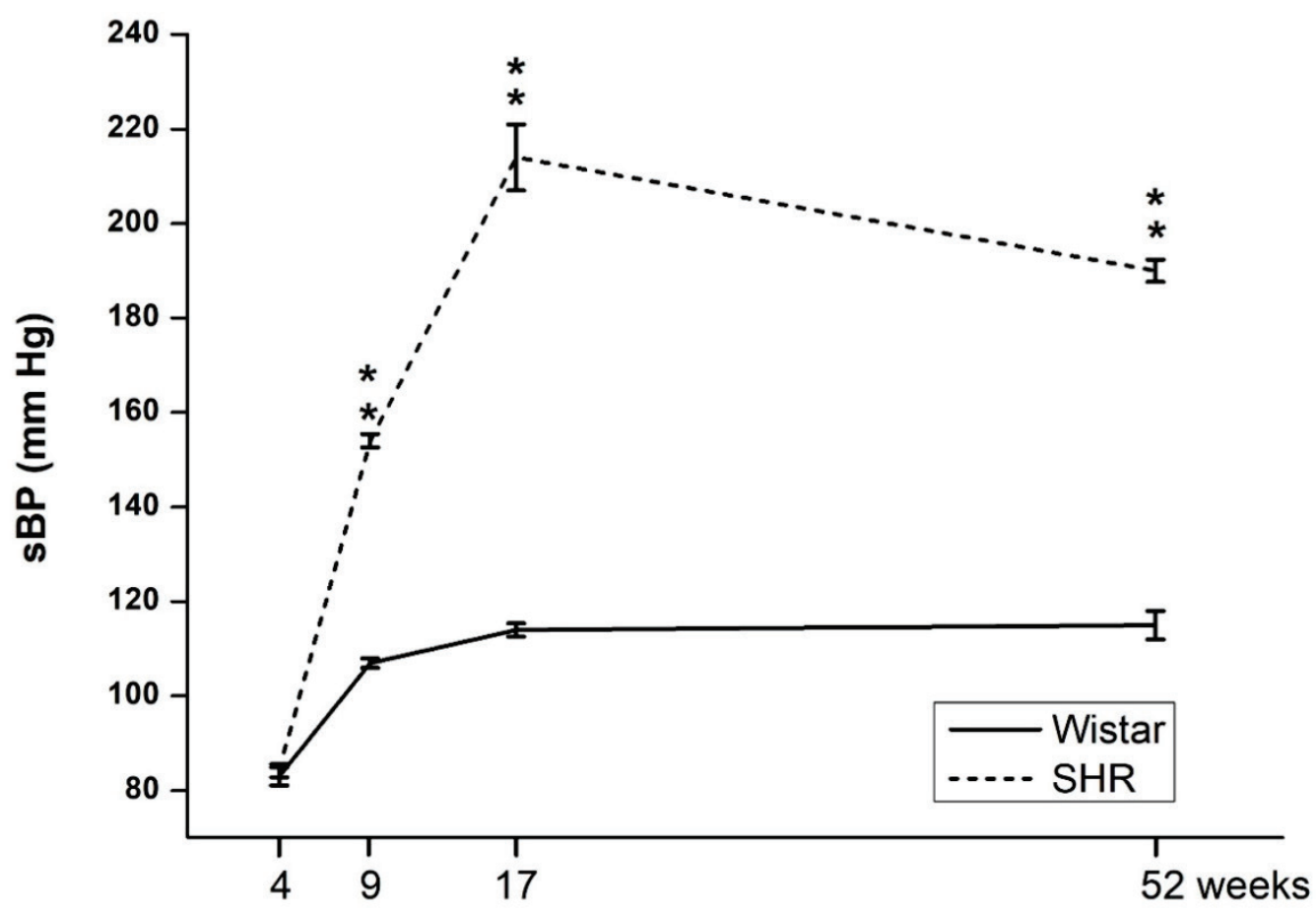

Fig. 1. Systolic blood pressure of control Wistar rats and spontaneously hypertensive rats. The values represent the data at the end of $3,9,17$, and 52 weeks of age. Data are means S.E.M., $* * p<0.01$ vs. Wistar rats.

\section{Geometry of the thoracic aorta}

In 3-week-old SHRs, the values of all WT $(46.82 \pm 0.50 \mu \mathrm{m}), \quad \mathrm{CSA} \quad\left(132,700 \pm 2,220 \mu \mathrm{m}^{2}\right), \quad$ ID $(855 \pm 13 \mu \mathrm{m})$ and WT/ID $(0.0552 \pm 0.0011)$ of the thoracic aorta were significantly decreased $(\mathrm{p}<0.01)$ compared with those in normotensive Wistar rats $(63.53 \pm 1.28 \mu \mathrm{m}$, $203,300 \pm 5,440 \mu \mathrm{m}^{2}, \quad 954 \pm 15 \mu \mathrm{m}$, and $0.0668 \pm 0.0016$, respectively).

In 17-week-old SHRs, the increase $(\mathrm{p}<0.01)$ in WT $(69.03 \pm 2.66 \mu \mathrm{m}), \mathrm{CSA}\left(364,600 \pm 16,500 \mu \mathrm{m}^{2}\right)$, and $\mathrm{WT} / \mathrm{ID}$ ratio $(0.0429 \pm 0.0013)$ was observed compared to those in Wistar rats $\left(61.25 \pm 1.62 \mu \mathrm{m}, 308,900 \pm 6,200 \mu \mathrm{m}^{2}\right.$, and $0.0383 \pm 0.0011$, respectively);no difference between the ID in SHRs $(1,613 \pm 38 \mu \mathrm{m})$ and Wistar rats $(1,547 \pm 32 \mu \mathrm{m})$ was found.

In 52-week-old SHRs, an increase $(\mathrm{p}<0.01)$ in WT $(100.89 \pm 2.5 \mu \mathrm{m}), \operatorname{CSA}\left(703,200 \pm 22,300 \mu \mathrm{m}^{2}\right)$, and WT/ID $(0.0480 \pm 0.0025)$ was observed compared to that in age-matched Wistar rats $(64.68 \pm 0.99 \mu \mathrm{m}$, $463,000 \pm 8,255 \mu \mathrm{m}^{2}$, and $0.0325 \pm 0.0015$, respectively); no difference was observed between the ID $(2,109 \pm 37 \mu \mathrm{m})$ in SHRs and that in the same old Wistar rats $(2,100 \pm 44 \mu \mathrm{m})$.

Representative images of thoracic aorta and the values of WT, CSA, ID, and WT/ID in individual ontogenic periods of the thoracic aorta are illustrated in Figs 2, 3, 4, 5 and 6.

\section{Structure of the thoracic aorta}

The calculation of the CSAs of the three main components of the arterial wall (smooth muscle cells, extracellular matrix, and endothelial cells) based on their volume densities (proportional representation) in the thoracic wall revealed important discrepancies between SHRs and age-matched Wistar rats.

In 3-week-old SHRs, the masses (CSAs) of all SMCs $\left(53,000 \pm 1,300 \mu \mathrm{m}^{2}\right.$, it represents approximately $39.94 \%$ of arterial wall CSA), ECM $\left(77,000 \pm 6,500 \mu \mathrm{m}^{2}\right.$, $58.03 \%)$, and ECs $\left(2840 \pm 118 \mu \mathrm{m}^{2}, 2.14 \%\right)$ were significantly $(\mathrm{p}<0.01)$ decreased compared to those in Wistar rats $\left(86,000 \pm 1,600 \mu \mathrm{m}^{2}, \quad 42.30 \%\right.$, $114,000 \pm 7,300 \mu \mathrm{m}^{2}, \quad 56.08 \%$, and $3,710 \pm 281 \mu \mathrm{m}^{2}$, $1.83 \%$, respectively). 

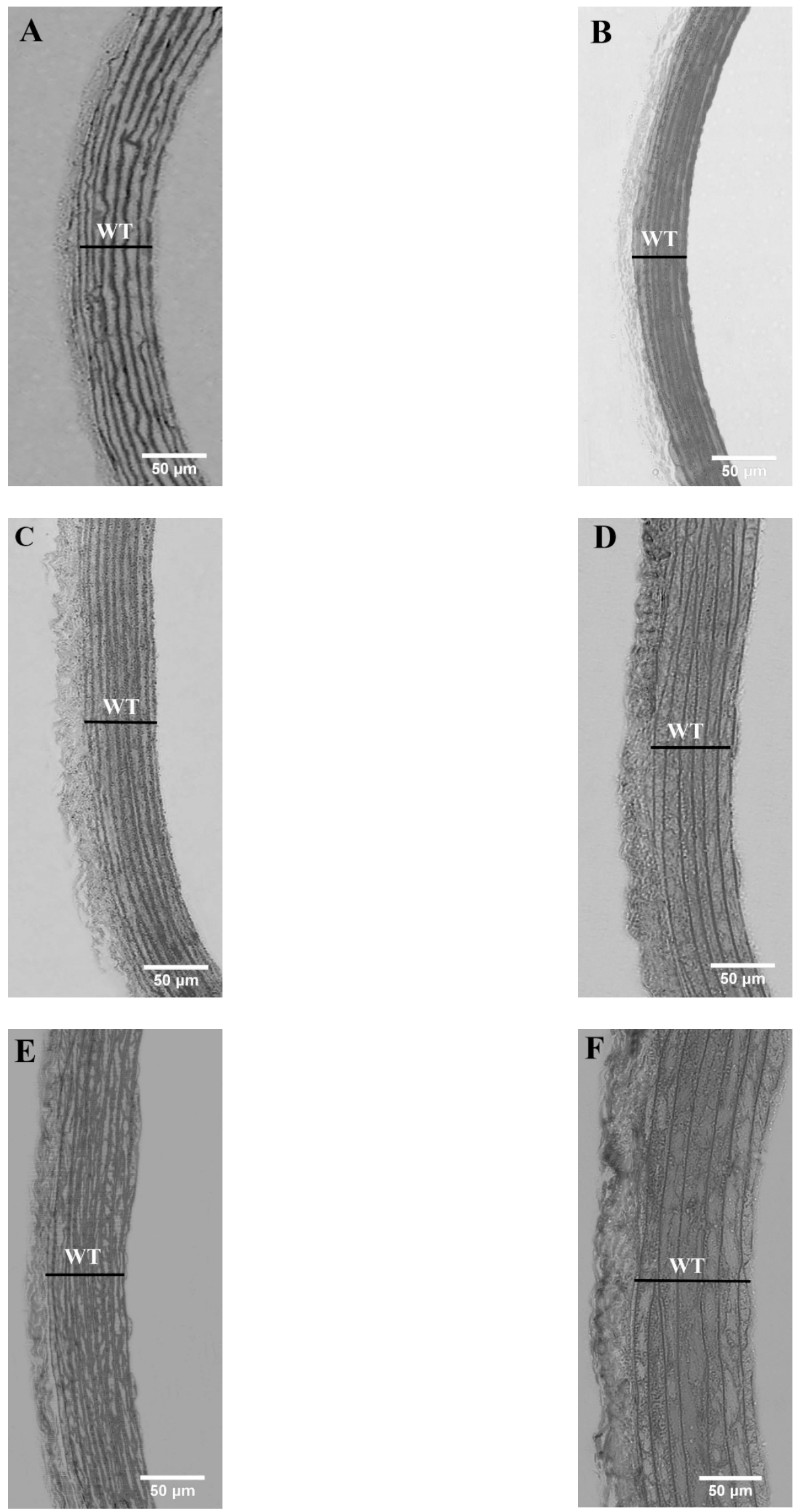

Fig. 2. Semi-thin sections of thoracic aorta. Wall thickness (WT) of Wistar rats in the age of 3 weeks (A), 17 weeks (C), and 52 weeks (E) and spontaneously hypertensive rats in the age of 3 weeks (B), 17 weeks (D), and 52 weeks (F). 


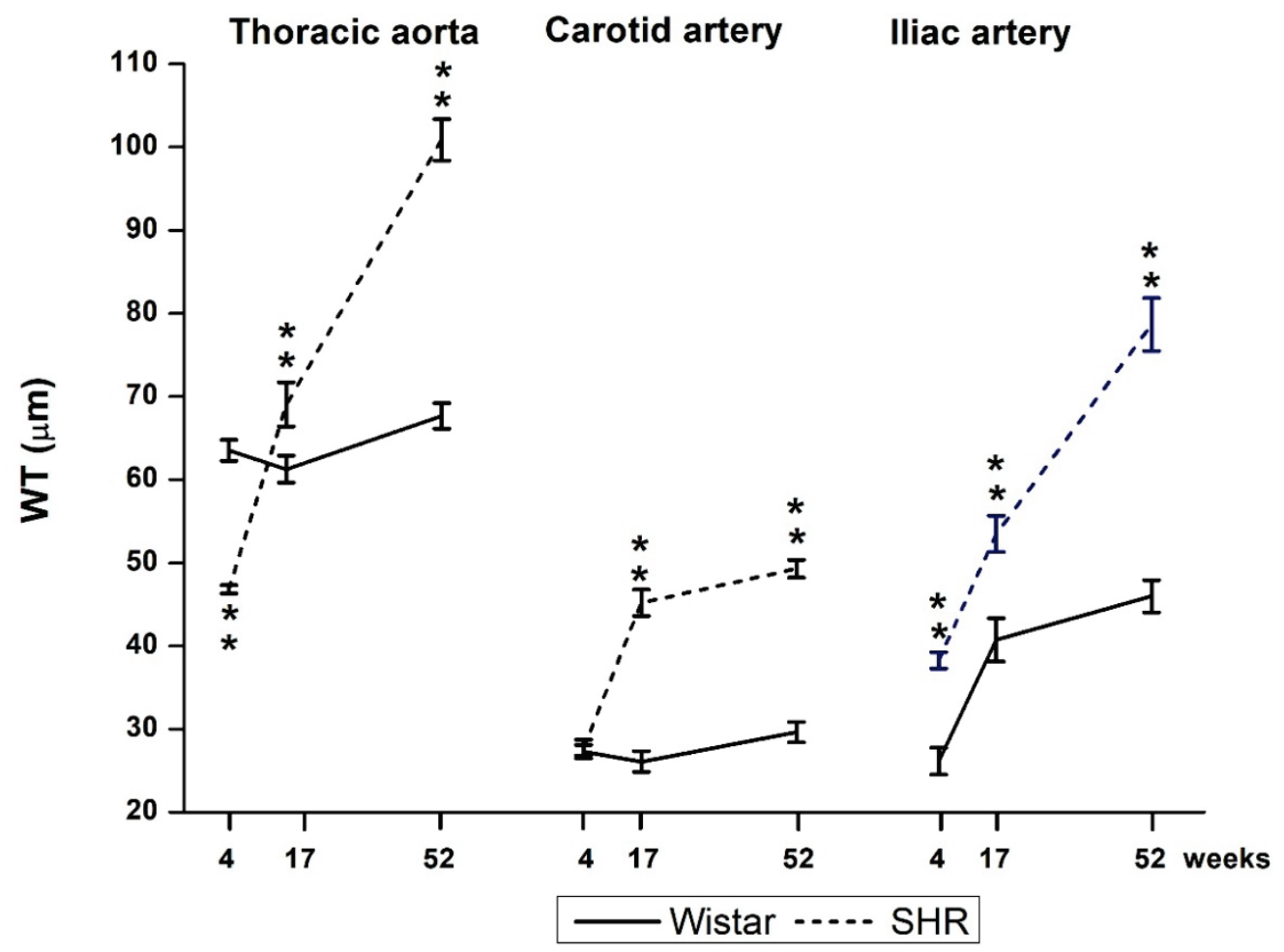

Fig. 3. Wall thickness - tunica intima+media (WT) of thoracic aorta, carotid artery, and iliac artery of Wistar rats and spontaneously hypertensive rats in the age of 3,17 , and 52 weeks. Data are means S.E.M. $* * p<0.01$ vs. Wistar rats.

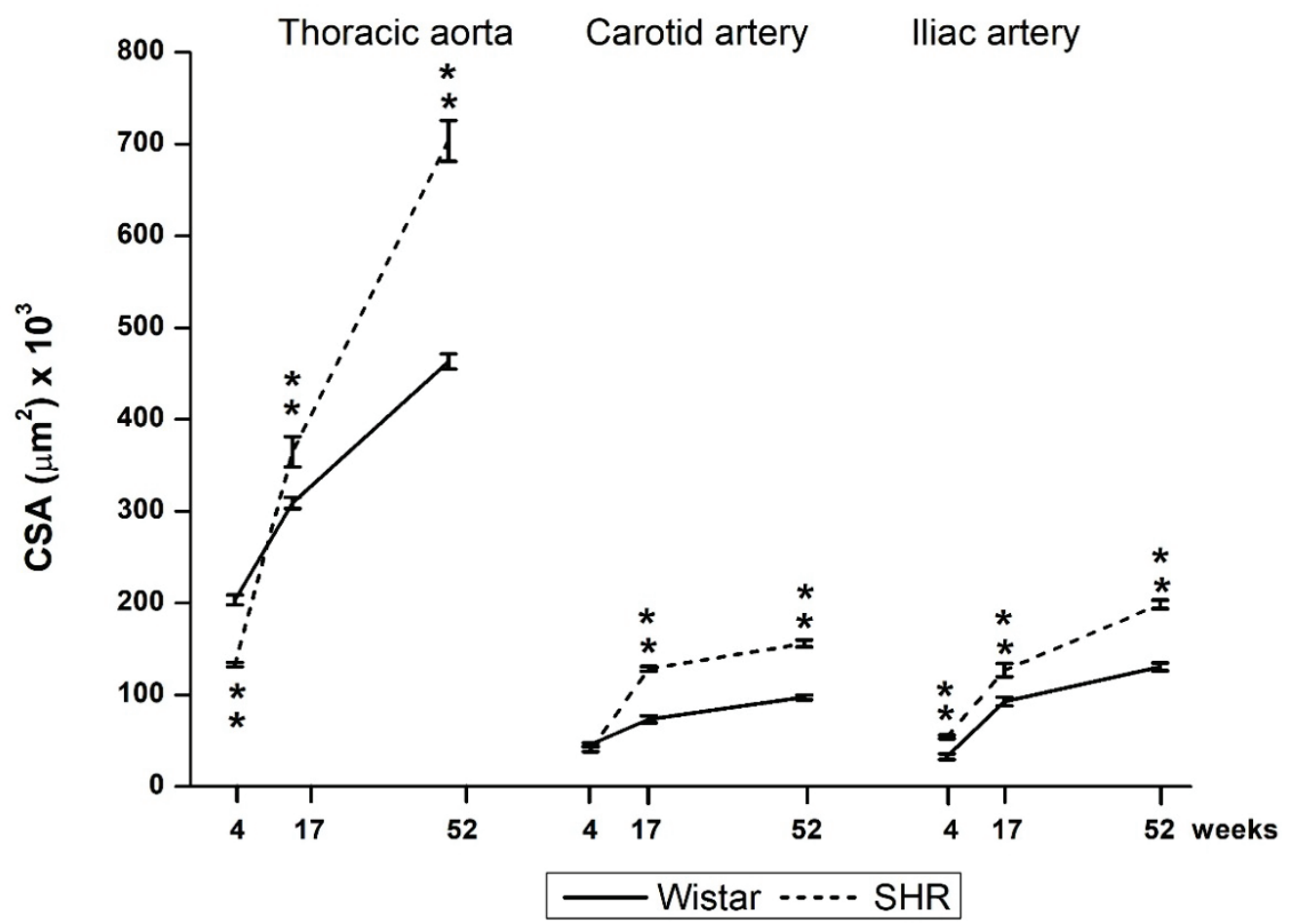

Fig. 4. Cross sectional area of the wall - tunica intima+media (CSA) of thoracic aorta, carotid artery, and iliac artery of Wistar rats and spontaneously hypertensive rats in the age of 3,17 , and 52 weeks. Data are means S.E.M. $* * p<0.01$ vs. Wistar rats. 


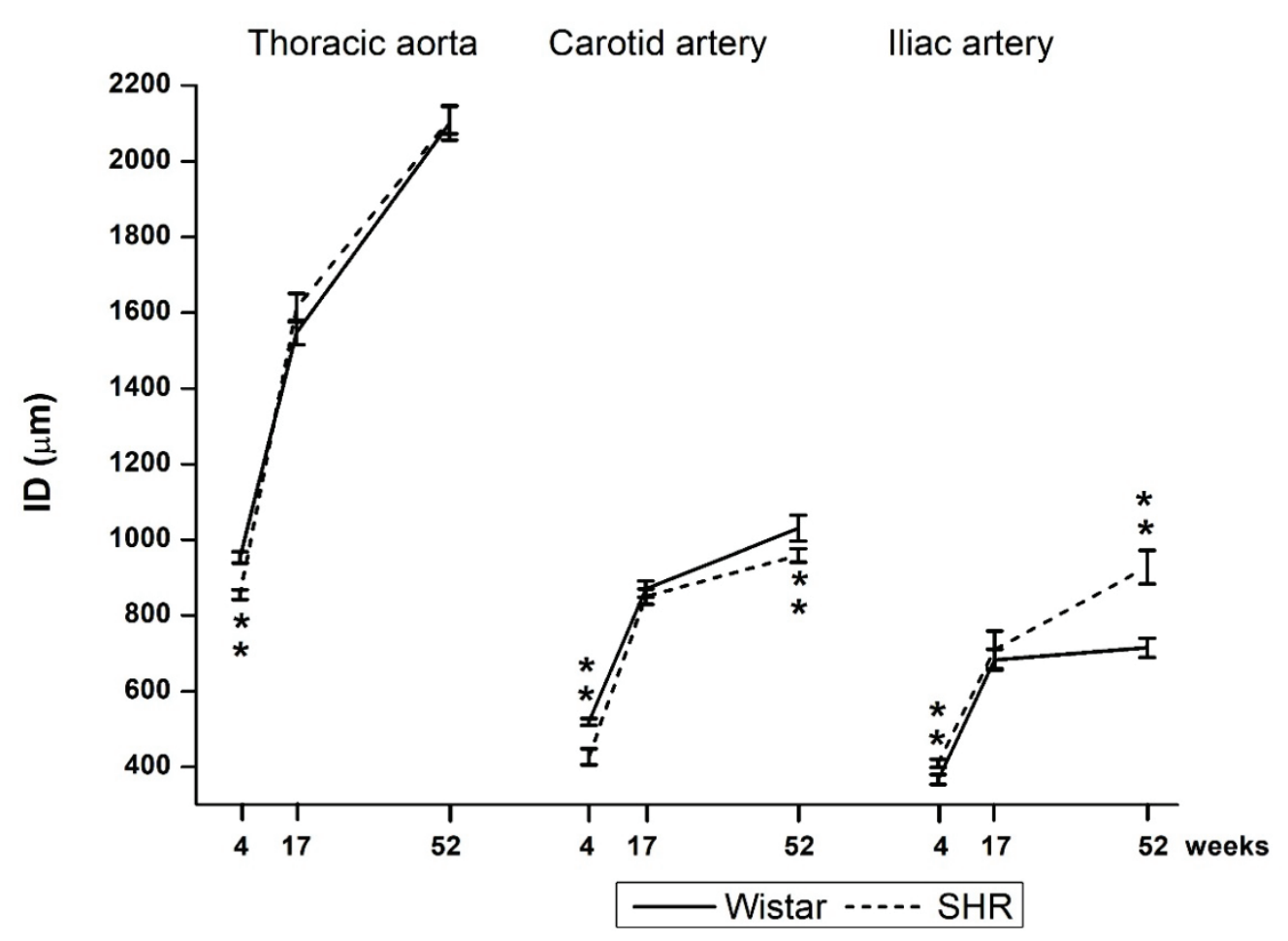

Fig. 5. Inner diameter (ID) of thoracic aorta, carotid artery, and iliac artery of Wistar rats and spontaneously hypertensive rats in the age of 3,17 , and 52 weeks. Data are means S.E.M. ${ }^{* *} p<0.01$ vs. Wistar rats.

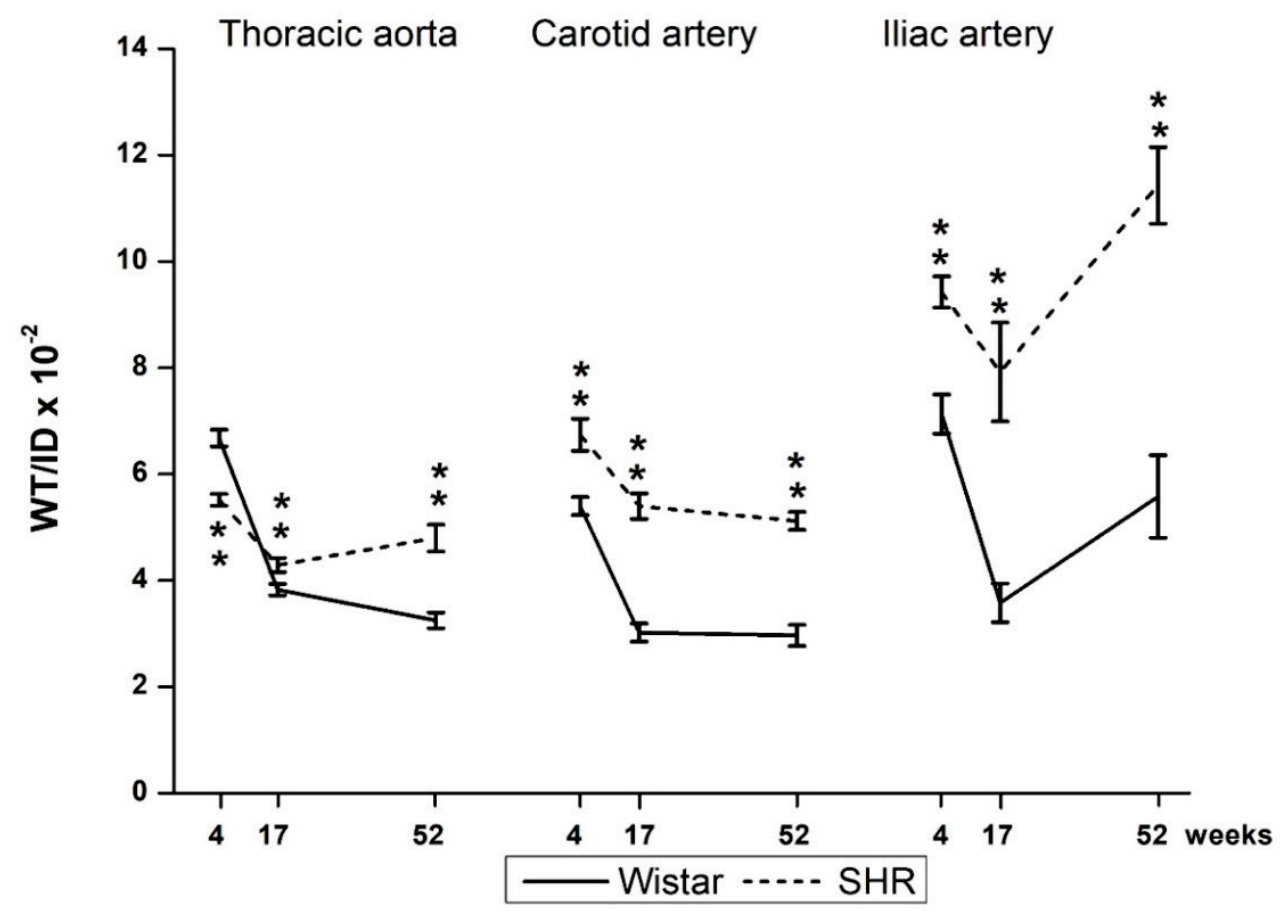

Fig. 6. Wall thickness/inner diameter (WT/ID) in thoracic aorta, carotid artery, and iliac artery of Wistar rats and spontaneously hypertensive rats in the age of 3,17 , and 52 weeks. Data are means S.E.M. ${ }^{* *} p<0.01$ vs. Wistar rats.

In 17-week-old SHRs, the masses (CSAs) of SMCs $\left(149,000 \pm 7,800 \mu \mathrm{m}^{2}, \quad 40.87 \%\right)$ and ECM $\left(206,000 \pm 5,500 \mu \mathrm{m}^{2}, 56.50 \%\right)$ were increased $(\mathrm{p}<0.01)$ compared with those in Wistar rats $\left(136,000 \pm 3,700 \mu \mathrm{m}^{2}\right.$, $\left.44.03 \%, \quad 159,000 \pm 3,700 \mu \mathrm{m}^{2}, \quad 51.47 \%\right), \quad$ and $\quad$ no difference in the CSA of ECs was observed between SHRs $\left(11,870 \pm 627 \mu \mathrm{m}^{2}, 3.26 \%\right)$ and Wistar rats $\left(13,480 \pm 1,495 \mu \mathrm{m}^{2}, 4.36 \%\right)$. 
In 52-week-old SHRs, the CSAs of SMCs $\left(306,000 \pm 13,600 \mu \mathrm{m}^{2}, \quad 43.52 \%\right), \quad$ ECM $\quad(368,000 \pm$ $\left.19,300 \mu \mathrm{m}^{2}, \quad 52.33 \%\right)$, and ECs $\left(29670 \pm 2437 \mu \mathrm{m}^{2}\right.$, $4.22 \%$ ) were significantly $(\mathrm{p}<0.01)$ elevated compared to those in Wistar rats $\left(206,000 \pm 10,900 \mu \mathrm{m}^{2}, 44.49 \%\right.$,

A

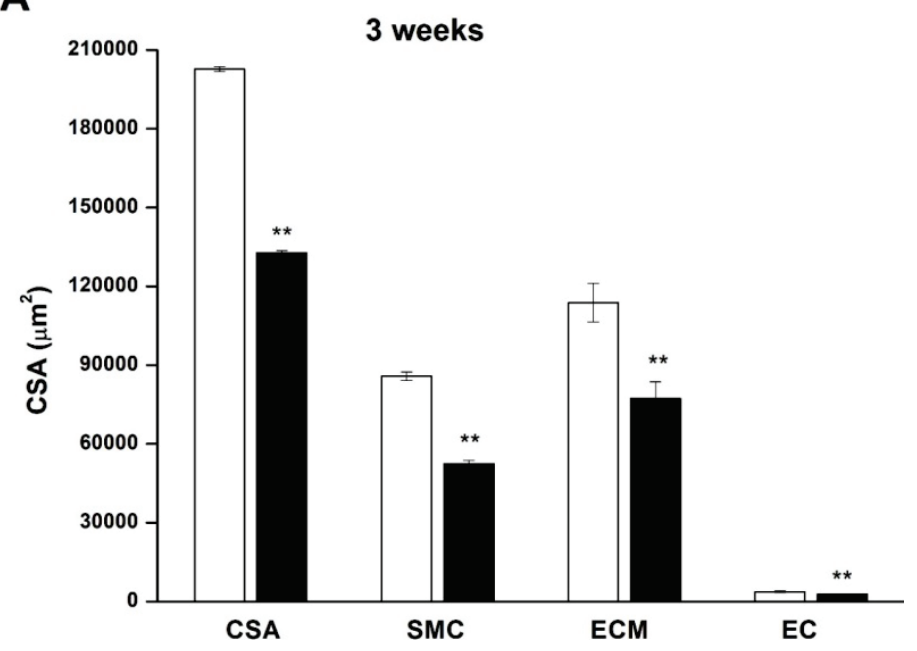

B

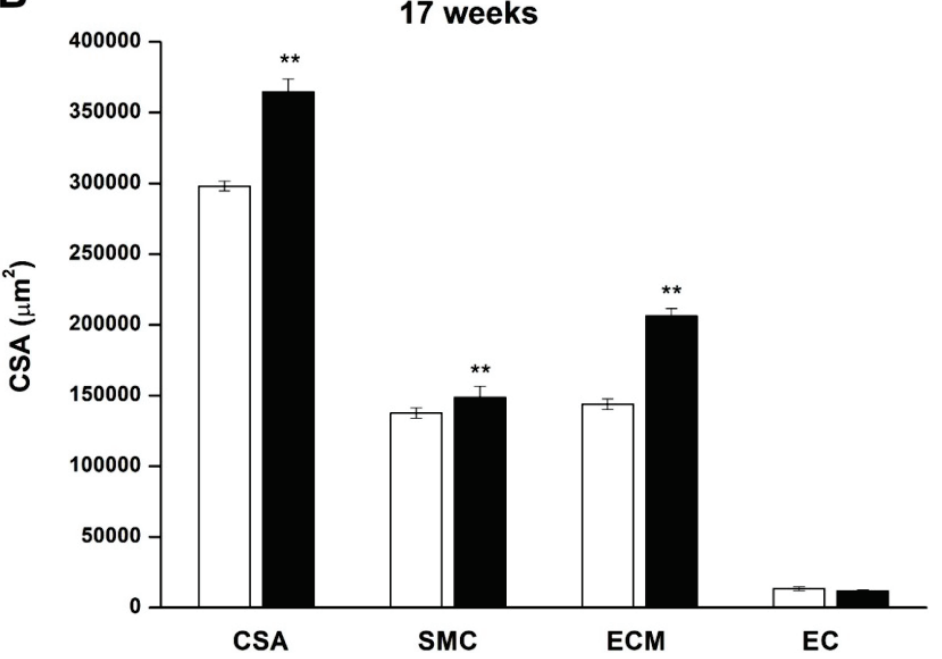

C

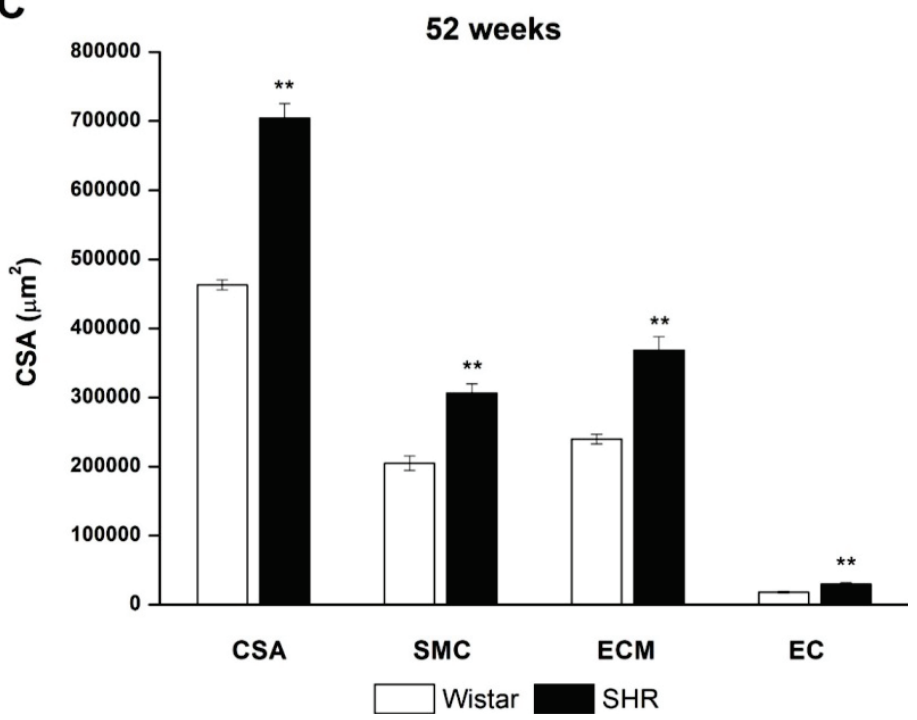

Fig. 7. Total cross sectional area - tunica intima+media (CSA), cross sectional area of smooth muscle cells (SMC), extracellular matrix in tunica intima+media (ECM), and endothelial cells (EC) based on volume densities in thoracic aorta of Wistar rats (Wistar) and spontaneously hypertensive rats (SHR) in the age of 3 weeks (A), 17 weeks (B), and 52 weeks (C). Data are means S.E.M. ${ }^{* *} \mathrm{p}<0.01$ vs. Wistar rats. 
Geometry of the carotid artery

In 3-week-old SHRs, no differences were observed in the WT $(27.77 \pm 0.97 \mu \mathrm{m})$ and CSA $\left(40,410 \pm 2,670 \mu^{2}\right) \quad$ compared with those in normotensive Wistar rats $(27.27 \pm 0.80 \mu \mathrm{m}$, $46,150 \pm 1,900 \mu \mathrm{m}^{2}$, respectively). The ID in SHRs $(427 \pm 21 \mu \mathrm{m})$ was decreased $(\mathrm{p}<0.01)$, and the WT/ID $(0.0674 \pm 0.0030)$ was increased $(p<0.01)$, compared to those in Wistar rats $(519 \pm 9 \mu \mathrm{m}$ and $0.054 \pm 0.0017$, respectively).

In 17-week-old SHRs, a pronounced increase $(\mathrm{p}<0.01) \quad$ in the WT $(45.19 \pm 1.60 \mu \mathrm{m}), \quad$ CSA $\left(128,130 \pm 2,580 \mu \mathrm{m}^{2}\right)$, and WT/ID $(0.0540 \pm 0.0024)$ was found compared to that in Wistar rats $(26.08 \pm 1.24 \mu \mathrm{m}$, $73,300 \pm 3,620 \mu \mathrm{m}^{2}$, and $0.0302 \pm 0.0017$, respectively). No difference in the ID between SHRs $(850 \pm 20 \mu \mathrm{m})$ and Wistar rats $(870 \pm 21 \mu \mathrm{m})$ was found.

In 52-week-old SHRs, an increase $(\mathrm{p}<0.01)$ in the WT $(49.29 \pm 1.08 \mu \mathrm{m}), \operatorname{CSA}\left(155,850 \pm 3,860 \mu \mathrm{m}^{2}\right)$, and WT/ID $(0.0512 \pm 0.0017)$ was found compared to that in Wistar rats $\left(29.64 \pm 1.23 \mu \mathrm{m}, 97,170 \pm 2,650 \mu \mathrm{m}^{2}\right.$, and $0.0297 \pm 0.0020$, respectively). No difference between ID in SHRs $(959 \pm 18)$ and Wistar rats $(1,031 \pm 34)$ was found.

The values of WT, CSA, ID, and WT/ID in individual ontogenic periods of the carotid artery are illustrated in Figures 3, 4, 5 and 6.

\section{Geometry of the iliac artery}

In 3-week-old SHRs, the values of the WT $(38.26 \pm 1.00 \mu \mathrm{m}), \mathrm{CSA}\left(54,070 \pm 2,100 \mu \mathrm{m}^{2}\right)$, ID $(410 \pm$ $10.79 \mu \mathrm{m})$, and WT/ID $(0.0943 \pm 0.0029)$ were increased $(\mathrm{p}<0.01)$, compared with those in Wistar rats WT 26.13 \pm $1.62 \mu \mathrm{m}, \quad$ CSA $32,510 \pm 2,900 \mu \mathrm{m}^{2}, \quad$ WT/ID $\quad(0.0713 \pm$ $0.0037)$, and ID $(367 \pm 12.87 \mu \mathrm{m})$.

In 17-week-old SHRs, the WT $(53.48 \pm 2.18 \mu \mathrm{m})$, CSA $\left(126,800 \pm 7,340 \mu \mathrm{m}^{2}\right)$, and WT/ID $(0.0792 \pm 0.0093)$ were increased $(\mathrm{p}<0.01)$ compared to those in Wistar rats (40.72 $\left.\pm 2.6 \mu \mathrm{m}, 92,540 \pm 4,640 \mu \mathrm{m}^{2}, 0.0358 \pm 0.0036\right)$. No difference was observed between the ID $(710 \pm 49.22 \mu \mathrm{m})$ of SHRs and ID $(683 \pm 27.53 \mu \mathrm{m})$ of Wistar rats.

In 52-week-old SHRs, all of the parameters studied $\quad-\quad$ WT $\quad(78.66 \pm 3.17 \mu \mathrm{m}), \quad$ CSA $\left(198,700 \pm 4.72 \mu \mathrm{m}^{2}\right)$, ID $(928 \pm 44.36 \mu \mathrm{m})$, and WT/ID $(0.114 \pm 0.0072)$ - were increased $(\mathrm{p}<0.01)$ compared with those in normotensive Wistar rats $(45.98 \pm 1.93 \mu \mathrm{m}$, $130,400 \pm 4,410 \mu \mathrm{m}^{2}, \quad 715 \pm 25.36$, and $0.0558 \pm 0.0078$, respectively).

The values of the WT, CSA, ID, and WT/ID in individual ontogenic periods of carotid artery are illustrated in Figures 3, 4, 5 and 6.

\section{Discussion}

The experiments revealed that the values of sBP between SHRs and Wistar rats did not significantly differ at the age of 3 weeks. From the $5^{\text {th }}$ week of age, the sBP was increased in both Wistar rats and SHRs; however, in SHRs, the increase was more rapid than that in Wistar rats. Moreover, in the $6^{\text {th }}$ week of age and during the whole course of the experiment, the sBP was significantly elevated in SHRs compared with that in Wistar rats. The findings are in accordance with our repeatedly published data (Kristek 2000, Cebova and Kristek 2011, Kristek et al. 2013) and the observations of others (Cosson et al. 2007, Dickhout and Lee 1997, Van Gorp et al. 2000), including the original data published by Okamoto and Aoki (1963).

The evaluation of the trophicity (CSAs) of the thoracic aorta, as well as the carotid, iliac, coronary (Cebova and Kristek 2011), and large mesenteric (Dickhout and Lee 2000) arteries in SHRs revealed pronounced quantitative differences between individual arteries of normotensive rats and SHRs during ontogeny. Pronounced alterations in the SHR arteries were already found in the prehypertensive period.

We found that the wall thickness of the thoracic aorta and its CSA in the prehypertensive period were surprisingly lower in SHRs than in age-matched Wistar rats. In the carotid and coronary arteries (Cebova and Kristek 2011), no differences in this respect were observed between SHRs and Wistar rats. In the iliac artery and large mesenteric artery (Dickhout and Lee 2000), even hypertrophy of the arterial wall was observed in 3- to 4-week-old SHRs compared to that in normotensive rats. Cunha et al. (1997) observed an increase in the wall thickness of the carotid artery in 5-week-old SHRs. Van Gorp et al. (2000) found a decrease in the CSA of the thoracic wall (absolute value) in 3-month-old SHRs compared to WKY rats, although the CSA was increased in 1.5-month-old SHRs, and no difference was found in 6-month-old SHRs. Chamiot-Clerc et al. (2001) observed an increase in the CSA of the thoracic aorta already from 3-week-old SHR compared to that in WKY rats. Unfortunately, they did not mention the body weight of the young animals. We suggest that the differences between our and their results could also be evoked by the different methodology used in the experiments. The WT and CSA of the thoracic 
aorta in SHRs reached the control values at about the $9^{\text {th }}$ week of age, but the WT and CSA in the carotid and coronary (Cebova and Kristek 2011, Kristek et al. 2013) arteries were already significantly increased (at this period). In the $17^{\text {th }}$ week of age, the WTs and CSAs were significantly elevated in all arteries compared to those in Wistar rats. Nevertheless, the increment of CSAs compared to that in age-matched Wistar rats was disproportional among the arteries. In the $17^{\text {th }}$ week, it represented approximately $25 \%$ in the thoracic aorta, $37 \%$ in the iliac artery, $75 \%$ in the carotid artery, and approximately $260 \%$ in the coronary artery (Cebova and Kristek 2011, Kristek et al. 2003). In the next ontogeny period (52 weeks), the WT and CSA continued to increase in all arteries, a finding that was also documented in other types of arteries (Clozel et al. 1989). In normotensive animals, the wall thickness of the thoracic aorta, and carotid and coronary (Cebova and Kristek 2011) arteries (not iliac artery) did not change in the course of ontogeny despite the increase in body weight (the body weight increased from 3-4 weeks to 52 weeks of age by nearly ten times). In SHR wall thickness increased in harmony with BP elevation.

The inner diameter in both strains was continuously enlarged during ontogeny in concert with blood pressure elevation. The difference between normotensive rats and SHRs was found only in the prehypertensive period. In 3-week-old SHRs, the diameters of the aorta, and carotid and iliac arteries were smaller than those in Wistar rats; at about the $9^{\text {th }}$ week of age, the diameters reached the control values and did not differ from the controls during the next ontogeny period despite the different increments in BP elevation. In the coronary artery, the inner diameter continuously increased during the entire ontogeny (Cebova and Kristek 2011). We suggest that it could be evoked by different filling of the coronary artery compared to that of other arteries. Because the inner diameter in both strains increases concomitantly with age, we suppose that the inner diameter is determined beside the BP value also by the body weight. Thus, the decrease in the inner diameter in the thoracic aorta and carotid artery in the prehypertensive period in SHRs should be due to the decrease in the body weight. We suggest that, in later periods, this "body weight effect" in SHRs (they have a lower body weight in the whole experiment) could be compensated by a significant BP increase. Because the wall thickness of the thoracic aorta, and the carotid and coronary (Cebova and Kristek 2011) arteries in normotensive animals did not change in the course of ontogeny, the increase in the CSAs in Wistar rats was performed mainly due to the increment in the inner diameter. In SHRs, contrary to those in Wistar rats, both the wall thickness and inner diameter in the above arteries were enlarged and jointly contributed to the increase in the arterial wall mass during ontogeny.

The relationship among the ID, WT and BP is important because they determine the circumferential stress $(\mathrm{BP} \times \mathrm{ID} / \mathrm{WT})$ that participates in the remodeling of the arterial wall (Thubricar and Robiscek 1995). Considering LaPlace law, the WT increase in the SHR should be a compensatory effect against the increasing circumferential stress due to increased BP. A pronounced decrease in the WT/ID ratio in the aorta (compared to other arteries in SHRs and arteries of Wistar rats) in the prehypertensive period resulted in the increase in the circumferential stress on the arterial wall. The increased circumferential stress together with disorder in the wall structure could affect arterial wall stiffening, pulse wave, and cushioning function and could have a deleterious effect on the heart that can lead to hypertrophy of the heart in the prehypertensive period. Nevertheless, there is an open question regarding why TA is hypotrophic in prehypertensive period, and their branches are normotrophic or hypertrophic. In the muscular type of arteries, several authors have observed no structural changes or hypertrophy in the early ontogeny period in SHRs (Dickhout and Lee 1997, Rizzoni et al. 2000). In contrast, what was seen in the conduit arteries of SHRs (no changes or increase in ID) was observed in the small arteries. Most of the experiments confirmed a decrease in the inner diameter together with remodeling of the arterial wall in small arteries in long-term hypertension (Dickhout and Lee 1997, Mulvany et al. 1996).

The calculation of the CSAs of endothelial and muscle cells and extracellular matrix in TA based on their volume densities (proportional representation) proved that the mass (CSAs) of these components in the arterial wall was increased in both strains during ontogeny but with different intensities. Quantitative analysis revealed that hypotrophy of the thoracic aorta observed in the prehypertensive period of SHRs is evoked by a decreased mass of all the above components of the arterial wall. While the CSA of the smooth muscle and CSA of the extracellular matrix in the aorta reached or overcame the control value between the $9^{\text {th }}$ and $17^{\text {th }}$ weeks of age, the CSA of endothelial cells (absolute value) was in the $17^{\text {th }}$ week of age, still below the control value. 
In 52-week-old SHR, the CSAs of all components significantly overcame the control values. In contrast to the aorta in carotid artery (Kristek 2000), the CSA of all components was significantly increased in 9-week-old SHRs compared to that in Wistar rats.

The morphological findings are in good agreement with the results of functional studies. Contractile responses of the thoracic aorta to noradrenaline compared to that of Wistar rats were decreased in the prehypertensive period of SHRs (Torok et al. 2006, Zemancikova and Torok 2013). Because the sensitivity of smooth muscle cells to noradrenaline was not changed during this ontogeny period (Cacanyiova et al. 2016), we suggest that the decreased contractile force of the thoracic aorta could be due to hypotrophy of the smooth muscle. Diminished contractile responses of the thoracic aorta persist until approximately 12 weeks of age. Flexibility of the arterial wall depends on the harmony between endothelial functions and smooth muscle responsiveness. We suggest that decreased contractility and endothelial hypotrophy in the early ontogeny periods could be an inherent adaptive mechanism. Despite the decreased mass of EC in TA until adulthood, SHRs are very probably not NO deficient. Most authors suggest the overproduction of NO as a compensatory mechanism against blood pressure increase (Ulker et al. 2003). Likewise, the chronic increase of the NO level due to the long-term administration of NO donors did not evoke a beneficial effect on the arterial structure in adult SHRs (Kristek et al. 2003). Moreover, a fully functional NO system has been observed in spontaneously hypertensive rats already in the prehypertensive period (Gerova et al. 2002, Puzserova et al. 2014) and adult (28 weeks old) SHRs (Ulker et al. 2003). Nevertheless, in SHRs, the effectivity of NO can be decreased by the overproduction of superoxides (Tang et al. 2007). We suggest that the hypotrophy of ECs in early ontogeny and in young SHRs
( 3 and 9 weeks old) could be one of the reasons for the ambiguity of endothelium-dependent relaxations of the thoracic aorta to acetylcholine (Ajay and Mustafa 2006, Behr-Roussel et al. 2005), especially in experiments using SHRs of variable age. The decreased mass of ECs observed only in TA and due to this possible (if any) decrease in endogenous NO production in aorta could be replaced by ECs of other conduit arteries or from other sources e.g. by NO-synthase-independent nitrate production (Zhao et al. 2012) or with the ability of endothelial cells to release vasoactive compounds such as hydrogen sulphide.

In summary. The study revealed differences in the remodeling of the conduit arteries during the development of hypertension in SHRs. In prehypertensive period of SHR (3 weeks of the age) hypotrophy of TA, normotrophy of CA, and hypertrophy of IA was observed. Thus, it is impossible to exactly predict the consequences in an individual arterial wall evoked by blood pressure changing and vice versa. Along with blood pressure elevation arterial wall area increases and in adult SHR (17 and 52 weeks old) it was hypertrophied in all arteries studied. We suggest that different modes of alteration during the ontogeny of SHRs (from the prehypertensive period to late adulthood) reflect the flexibility of individual parts of the arterial tree to the different needs of the supplied areas.

\section{Conflict of Interest}

There is no conflict of interest.

\section{Acknowledgements}

Financial support by Slovak grants VEGA 2/0074/14, 2/0048/17, Ministry of Health of the Slovak Republic under the project registration number 2012/51-SAV-1 and APVV-15-0565 is gratefully acknowledged. We thank L. Kosnacova for her technical assistance.

\section{References}

AJAY M, MUSTAFA MR: Effects of ascorbic acid on impaired vascular reactivity in aortas isolated from age-matched hypertensive and diabetic rats. Vasc Pharm 45: 127-133, 2006.

AVOLIO AP, BUTLIN M, WALSH A: Arterial blood pressure measurement and pulse wave analysis-their role in enhancing cardiovascular assessment. Physiol Meas 31: R1-R47, 2010.

BEHR-ROUSSEL D, GORNY D, MEVEL K, COMPAGNIE S, KEM P, SIVAN V, BERNABE J, BEDIGIAN MP, ALEXANDRE L, GIULIANO F: Erectile dysfunction: an early marker for hypertension? A longitudinal study in spontaneously hypertensive rats. Am J Physiol 288: R276-R283, 2005. 
CACANYIOVA S, BERENYIOVA A, KRISTEK F, DROBNA M, ONDRIAS K, GRMAN M: The adaptive role of nitric oxide and hydrogen sulphide in vasoactive responses of thoracic aorta is triggered already in young spontaneously hypertensive rats. J Physiol Pharmacol 67: 501-512, 2016.

CEBOVA M, KRISTEK F: Age-dependent ultrastructural changes of coronary artery in spontaneously hypertensive rats. Gen Physiol Biophys 30: 364-372, 2011.

CLOZEL JP, KUHN H, HEFTI F: Decreases of vascular hypertrophy in four different types of arteries in spontaneously hypertensive rats. Am J Med 87 (6B): 92S-95S, 1989.

COSSON E, HERISSE M, LAUDE D, THOMAS F, VALENSI P, ATTALI JR, SAFAR ME, DABIRE H: Aortic stiffness and pulse pressure amplification in Wistar-Kyoto and spontaneously hypertensive rats. Am J Physiol Heart Circ Physiol 292: H2506-H2512, 2007.

CUNHA RS, DABIRE H, BEZIE I, WEISS AM, CHAOUCHE-TEYARA K, LAURENT S, SAFAR ME, LACOLLEY P: Mechanical stress of the carotid artery at the early phase of spontaneous hypertension in rats. Hypertension 29: 992-998, 1997.

DICKHOUT JG, LEE RM: Increased medial smooth muscle cell length is responsible for vascular hypertrophy in young hypertensive rats. Am J Physiol 279: H2085-H2094, 2000.

DICKHOUT JG, LEE RM: Structural and functional analysis of small arteries from young spontaneously hypertensive rats. Hypertension 29: 781-789, 1997.

GEROVA M, BERNATOVA I, TOROK J, JURANI M: Cardiovascular system of offsprings of hypertensive rats with defective nitric oxide production. Physiol Res 51: 465-474, 2002.

CHAMIOT-CLERC P, RENAUD JF, SAFAR ME: Pulse pressure, aortic reactivity, and endothelium dysfunction in old hypertensive rats. Hypertension 37: 313-321, 2001.

KRISTEK F, FABEROVA V, VARGA I: Long-term effect of molsidomine and pentaerythrityl tetranitrate on cardiovascular system of spontaneously hypertensive rats. Physiol Res 52: 709-717, 2003.

KRISTEK F, MALEKOVA M, CACANYIOVA S: Long-term effect of prazosin and losartan administration on blood pressure, heart, carotid artery, and acetylcholine induced dilation of cardiovascular system of young Wistar rats and SHR. Gen Physiol Biophys 32: 235-243, 2013.

KRISTEK F: Different structure of coronary wall in two types of hypertension: NO deficiency and SHR. In: The Biology of Nitric Oxide, Part 7. MONCADA S, GUSTAFSSON LE, WIKLUND NP, HIGGS EA (eds), Portland Press, London, 2000, p 69.

MULVANY MJ, BAUMBACH GL, AALKJAER C, HEAGERTY AM, KORSGAARD N, SCHIFFRIN EL, HEISTAD DD: Vascular remodeling. Hypertension 28: 505-506, 1996.

O'ROURKE MF, SAFAR ME: Relationship between aortic stiffening and microvascular disease in brain and kidney: cause and logic of therapy. Hypertension 46: 200-204, 2005.

OKAMOTO K, AOKI K: Development of a strain of spontaneously hypertensive rats. Jap Circ J 27: 282-293, 1963.

PUZSEROVA A, ILOVSKA V, BALIS P, SLEZAK P, BERNATOVA I: Age-related alterations in endothelial function of femoral artery in young SHR and WKY rats. BioMed Res Int 2014: 658479, 2014.

RIZZONI D, RODELLA L, PORTERI E, REZZANI R, GUELFI D, PICCOLI A, CASTELANO M, MUIESAN ML, BIANCHI R, ROSEI EA: Time course of apoptosis in small resistance arteries of spontaneously hypertensive rats. J Hypertens 18: 885-891, 2000.

SVIGLEROVÁ J, KUNCOVA J, NALOS L, TONAR Z, RAJDL D, STENGL M: Cardiovascular parameters in rat model of chronic renal failure induced by subtotal nephrectomy. Physiol Res 59 (Suppl 1): S81-S88, 2010.

TANG EH, LEUNG FP, HUANG Y, FELETOU M, SO KF, MAN RY, VANHOUTTE PM: Calcium and reactive oxygen species increase in endothelial cells in response to releasers of endothelium-derived contracting factor. Br J Pharmacol 151: 15-23, 2007.

THUBRIKAR MJ, ROBISCEK F: Pressure-induced arterial-wall stress and atherosclerosis. Ann Thorac Surg 59: 1594-1603, 1995.

TOROK J, KOPRDOVA M, CEBOVA M, KUNES J, KRISTEK F: Functional and structural pattern of arterial responses in hereditary hypertriglyceridemic and spontaneously hypertensive rats in early stage of experimental hypertension. Physiol Res 55 (Suppl 1): S65-S71, 2006. 
ULKER S, MCMASTER D, MCKEOWN PP, BAYRAKTUTAN U: Impaired activities of antioxidant enzymes elicit endothelial dysfunction in spontaneous hypertensive rats despite enhanced vascular nitric oxide generation. Cardiovasc Res 59: 488-500, 2003.

VAN GORP AW, SCHENAU DS, HOEKS AP, BOUDIER HA, DE MEY JG, RENEMAN RS: In spontaneously hypertensive rats alterations in aortic wall properties precede development of hypertension. Am J Physiol 278: H1241-H1247, 2000.

WEIBEL ER, KISTELER GS, SCHERLE WF: Practical stereological methods for morphometric cytology. J Cell Biol 30: 23-38, 1966.

ZEMANCIKOVA A, TOROK J: Diminished contractile responses of isolated conduit arteries in two rat models of hypertension. Chin J Physiol 56: 230-235, 2013.

ZHAO Y, VANHOUTTE PM, LEUNG SWS: Endothelial nitric oxide synthase-independent release of nitric oxide in the aorta of the spontaneously hypertensive rat. J Pharmacol Exp Ther 344: 15-22, 2012. 